Research Article

\title{
The Impact of Preconception TSH on the Reproductive Outcomes of Infertile Women Undergoing the First Fresh D3 Embryo Transfer Cycle
}

\author{
Yuchao Zhang $\mathbb{D}^{1}{ }^{1}$ Wenbin Wu $\mathbb{D}^{1},{ }^{1}$ Yanli Liu $\left(\mathbb{D},{ }^{1}\right.$ Yichun Guan $\mathbb{D}^{1},{ }^{1}$ Xingling Wang $\mathbb{D},{ }^{1}$ \\ and Liting Jia $\mathbb{C}^{2}$ \\ ${ }^{1}$ Department of Reproductive Medicine, The Third Affliated Hospital of Zhengzhou University, Zhengzhou, Henan, China \\ ${ }^{2}$ Neonatal Screening Center, The Third Affiliated Hospital of Zhengzhou University, Zhengzhou, Henan, China \\ Correspondence should be addressed to Xingling Wang; wangxl3661@163.com
}

Received 11 August 2020; Revised 23 October 2020; Accepted 3 November 2020; Published 12 November 2020

Academic Editor: Pawel Grzmil

Copyright (c) 2020 Yuchao Zhang et al. This is an open access article distributed under the Creative Commons Attribution License, which permits unrestricted use, distribution, and reproduction in any medium, provided the original work is properly cited.

\begin{abstract}
Purpose. To investigate the association between high-normal preconception TSH levels and reproductive outcomes in infertile women undergoing the first fresh D3 embryo transfer. Methods. This was a retrospective study. Euthyroid patients undergoing the first fresh D3 embryo transfer from January 2018 to May 2019 were initially included. The patients were divided into a low-TSH (0.27-2.5 mIU/L) group and a high-normal TSH (2.5-4.2 Miu) group. The reproductive outcomes were compared between the groups. Results. A total of 1786 women were ultimately included, in which 1008 of whom had serum TSH levels between 0.27 and $2.5 \mathrm{mIU} / \mathrm{L}$ and 778 of whom had serum TSH levels between 2.5 and $4.2 \mathrm{mIU} / \mathrm{L}$. The patients were highly homogeneous in terms of general characteristics. High-normal TSH levels had no adverse impact on the clinical pregnancy rate, miscarriage rate, or live birth rate (respectively, $\mathrm{aOR}=0.92,1.30$, and 0.88 and $P=0.416,0.163$, and 0.219 ). No significant differences were observed in terms of gestational age, single live birth rates, and birth weight, or birth length. Conclusion. High-normal TSH levels did not significantly influence reproductive outcomes in infertile women undergoing the first fresh D3 embryo transfer. Further studies are needed to test whether the results might be applicable to a wider population.
\end{abstract}

\section{Introduction}

Subclinical hypothyroidism (SCH), which was common in clinical practise, was a subtle deficiency in thyroid function regardless of the elevated thyrotropin (TSH) level and normal free thyroxine (FT4) level [1]. There is still debate regarding the definition of $\mathrm{SCH}$ (the threshold value might range from 2.5 to $5.5 \mathrm{mIU} / \mathrm{L}$ according to different studies) and the decision of when to treat, particularly for women attempting pregnancy [2]. When $\mathrm{SCH}$ was defined as a TSH level $>4.0 \mathrm{mIU} / \mathrm{L}$, there was fair evidence showing that it was associated with miscarriage and adverse obstetric outcomes. Experts suggested that TSH concentrations $<2.5 \mathrm{mIU} / \mathrm{L}$ should be maintained during the ART procedure in infertile patients. However, there is insufficient evidence indicating an association between high-normal preconception TSH
(2.5-4.0 mIU/L) and the reproductive outcomes [3]. We previously investigated the impact of high-normal preconception TSH on the reproductive outcomes of infertile women undergoing their first IUI treatment and observed no association [4]. Similar results were also observed in women undergoing the first single fresh D5 blastocyst transfer. However, different opinions were held by other instigators as various confounders (such as the transfer of cleavage embryo or blastocysts, the number and quality of the transferred embryo, and the age and ethnicity of included women) influenced the reproductive outcomes [5-14]. For that reason, the aim of this study was to further evaluate the impact of preconception TSH levels between 2.5 and $4.2 \mathrm{mIU} / \mathrm{L}$ (which was used as the upper limit of the reference range in our laboratory) on reproductive outcomes in women undergoing their first fresh D3 embryo transfer. 
1.1. Patients. A total of 2542 infertile women receiving fresh D3 embryo transfer in the Department of Reproductive Medicine, the Third Affiliated Hospital of Zhengzhou University, from January 2018 to May 2019, were initially included. The women underwent standard controlled ovarian hyperstimulation $(\mathrm{COH})$ with a downregulation protocol using a GnRH-agonist or an antagonist protocol according to the patients' conditions. For women with poor ovarian response, mild ovarian stimulation was offered. Oocytes were retrieved transvaginally 34-36 h after HCG administration and were inseminated using either the IVF or the ICSI method. Rescued ICSI was performed when necessary to avoid failure of insemination. Variable cleavage embryos were observed and scored accordingly on the third day after insemination. High-quality embryos were preferentially transferred to the uterus under the guidance of ultrasound.

Patients with the following criteria were excluded: previous embryo transfers $(n=361)$; the presence of overt or subclinical thyroid dysfunction $(n=366)$; and lack of essential data $(n=29)$. After exclusions, 1786 women were transferred with fresh D3 embryos at the first IVF/ICSI cycle and were divided into two groups based on preconception TSH levels (low-TSH group, 0.27-2.5 mIU/L, $n=1008$; highnormal TSH group, 2.5-4.2 mIU/L, $n=778$ ). As euthyroid women with preconception $\mathrm{TSH}<4.2 \mathrm{mIU} / \mathrm{L}$ were included in this study, no further tests or treatments were provided to them after starting the ovarian stimulation. The study has been carried out in accordance with the Code of Ethics of the Declaration of Helsinki.

1.2. Laboratory Tests. The measurements of thyroid hormones were performed as previously described [4]. Generally, thyroid function tests were performed no longer than six months before starting the ovarian stimulation protocol. Daily internal quality control and yearly external quality control were carried out by request. The intra-assay coefficients of variation (CV) of serum TSH were $1.3 \%$, and the interassay $\mathrm{CV}$ value was $3.5 \%$.

1.3. Definition of Clinical Outcomes. Pregnancy was defined as positive serum $\beta$-hCG (>10 IU/L). Clinical pregnancy was defined as the presence of the gestational sac and fetal heart activity following positive serum $\beta$-hCG. Early embryo loss was defined as the lack of visible gestational sac or fetal heart activity following positive serum $\beta$-hCG. Ectopic pregnancy was defined as a pregnancy that did not occur inside the uterine cavity. Miscarriage, which included early abortion, intermediate abortion, and late abortion, was defined as a pregnancy that did not result in delivery. Preterm birth was defined as a gestational age less than 259 days (37 weeks). Live birth was defined as the delivery of live babies. Low live birth weight was defined as the neonatal weight less than $2500 \mathrm{~g}$.

1.4. Statistical Analyses. Our primary outcomes were clinical outcomes, such as clinical pregnancy rate, early pregnancy loss rate, miscarriage rate, and live birth rate. The secondary outcomes were obstetric outcomes, such as the single live birth rate, birth weight, birth length, and gestational age. Continuous data are expressed as the mean (SD), and statistical comparisons were performed by either Student's $t$ test or the Mann-Whitney $U$ test where appropriate. Categorical variables were expressed as numbers or percentages, and the chi-squared test or Fisher's exact test was used for comparisons of categorical variables where appropriate. Binary logistic regression was then used to evaluate the impact of preconception high-normal TSH levels on the clinical and obstetric outcomes. Data were analyzed using SPSS 22.0 statistical software. All $P$ values less than 0.05 were considered statistically significant.

\section{Results}

2.1. General Characteristics of the Included Women. The flowchart of the included women is shown in Figure 1. The results predicted that a cohort of highly homogeneous women was included. Specifically, there were no differences between the TSH groups in terms of age, BMI, AMH, FT4, endometrial thickness, duration of infertility, and basal FSH (Table 1). Furthermore, there were no differences in the frequencies of the causes or type of infertility between the two groups $(P=0.107,0.126)$. The frequencies of insemination methods and number and high-quality of transferred embryos were similar in both the groups $(P=0.172,0.669$, and 0.298 , respectively). No difference was noted in terms of the scheme of ovarian stimulation $(P=0.355)$.

2.2. Clinical Outcomes of the Women Undergoing the First Fresh D3 Embryo Transfer. As shown in Table 2, comparisons of pregnancy rate, clinical pregnancy rate, live birth rate, and single live birth rate were performed between the low-TSH and high-normal TSH groups. There were no significant differences in the abovementioned reproductive outcomes between the two groups. In addition, there were no significant differences in the early pregnancy loss rate, miscarriage rate, or ectopic pregnancy rate between the two groups. Furthermore, binary logistic regression analysis between the groups indicated the number of high-quality D3 embryos was positively associated with the clinical pregnancy and live birth, and that was negatively associated miscarriage with adjusted ORs of $1.73(P<0.001), 1.76$ $(P<0.001)$, and $0.66(P=0.002)$, respectively, while younger age had slightly but significantly positive effects on the clinical pregnancy, live birth, and miscarriage with adjusted ORs of $0.93(P<0.001), 0.91(P<0.001)$ and $1.12(P<0.001)$, respectively. Anyhow, high-normal TSH levels had no adverse impact on the abovementioned outcomes $(\mathrm{aOR}=0.92$, 0.88 , and 1.30 and $P=0.416,0.219$, and 0.163 for clinical pregnancy rate, live birth rate, and miscarriage rate, respectively) (Table 3 ).

2.3. Obstetric Outcomes of the Women Undergoing the First Fresh D3 Embryo Transfer. We further compared obstetric outcomes between the low-TSH and high-normal TSH groups. Of the 1786 women included, 471 in the low-TSH 


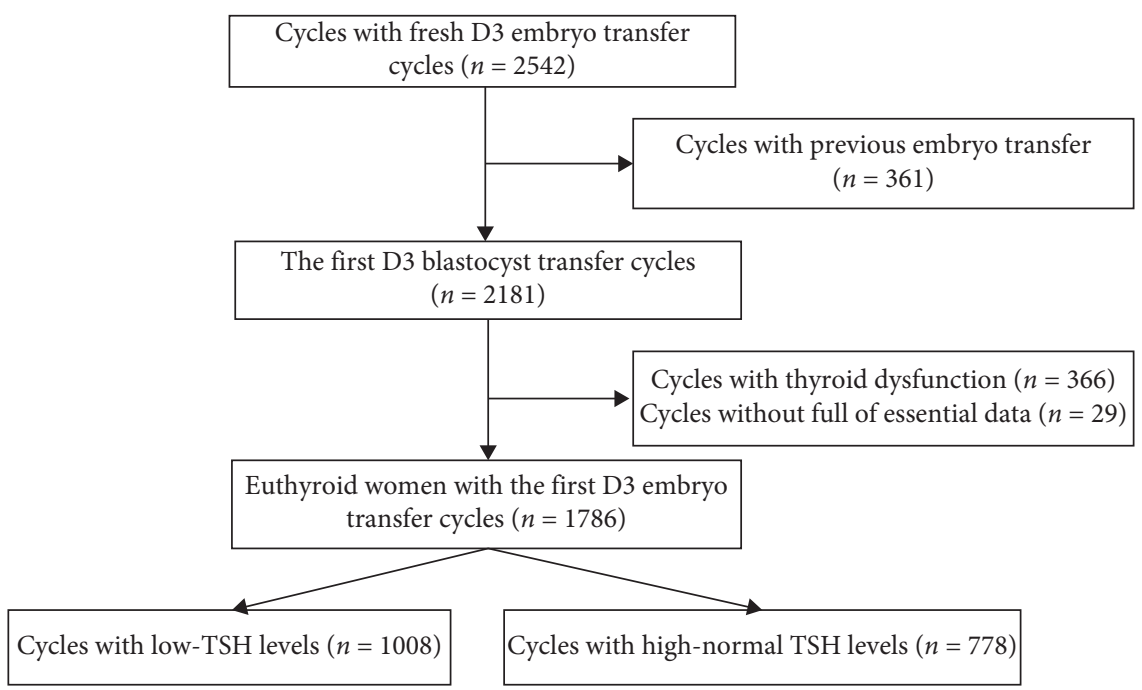

FIGURE 1: Flowchart of the inclusion of women undergoing the first fresh D3 embryo transfer cycles.

TABLE 1: The general characteristics of the included women based on TSH levels.

\begin{tabular}{|c|c|c|c|}
\hline & Low-TSH $(0.27-2.5 \mathrm{mIU} / \mathrm{L})$ & High-normal TSH (2.5-4.2mIU/L) & $P$ \\
\hline$N$ & 1008 & 778 & \\
\hline Age (years) & $31.82(4.75)$ & $31.62(4.59)$ & 0.371 \\
\hline BMI $\left(\mathrm{kg} / \mathrm{m}^{2}\right)$ & $23.71(3.27)$ & $23.98(3.38)$ & 0.088 \\
\hline $\mathrm{AMH}(\mathrm{pmol} / \mathrm{L})$ & $25.62(21.03)$ & $27.17(23.65)$ & 0.143 \\
\hline FT4 (pmol/L) & $16.77(6.81)$ & $16.51(5.56)$ & 0.385 \\
\hline FT3 (pmol/L) & $4.78(0.72)$ & $4.83(0.73)$ & 0.156 \\
\hline FSH (IU/L) & $7.52(2.32)$ & $7.67(2.3)$ & 0.169 \\
\hline Endometrial thickness (mm) & $11.29(2.31)$ & $11.46(2.37)$ & 0.138 \\
\hline Duration of infertility (year) & $3.52(2.68)$ & $3.68(2.78)$ & 0.212 \\
\hline \multicolumn{4}{|c|}{ Insemination methods of transferred embryos } \\
\hline ICSI & $246(24.4 \%)$ & $214(27.5 \%)$ & 0.172 \\
\hline IVF & $752(74.6 \%)$ & $552(71 \%)$ & \\
\hline IVF/ICSI & $10(1 \%)$ & $12(1.5 \%)$ & \\
\hline \multicolumn{4}{|l|}{ Ovarian stimulation protocol } \\
\hline GnRH agonist & $915(90.8 \%)$ & $720(92.5 \%)$ & 0.355 \\
\hline GnRH antagonist & $88(8.7 \%)$ & $56(7.2 \%)$ & \\
\hline Mild ovarian stimulation $^{\mathrm{a}}$ & $5(0.5 \%)$ & $2(0.3 \%)$ & \\
\hline \multicolumn{4}{|l|}{ Causes of infertility } \\
\hline Male factors (azoospermatism) ${ }^{\mathrm{b}}$ & $171(17 \%)$ & $159(20.4 \%)$ & 0.107 \\
\hline Male factors (others) & $56(5.6 \%)$ & $46(5.9 \%)$ & \\
\hline Female mixed factors & $38(3.8 \%)$ & $42(5.4 \%)$ & \\
\hline Ovulation failure & $70(6.9 \%)$ & $53(6.8 \%)$ & \\
\hline Pelvic and fallopian tube factors & $345(34.2 \%)$ & $259(33.3 \%)$ & \\
\hline Factors on both sides & $257(25.5 \%)$ & $160(20.6 \%)$ & \\
\hline Unexplained & $71(7 \%)$ & $59(7.6 \%)$ & \\
\hline \multicolumn{4}{|l|}{ Type of infertility } \\
\hline Primary & $545(54.1 \%)$ & $392(50.4 \%)$ & 0.126 \\
\hline Secondary & $463(45.9 \%)$ & $386(69.6 \%)$ & \\
\hline \multicolumn{4}{|l|}{ Number of transferred embryos } \\
\hline 1 & $110(10.9 \%)$ & $80(10.3 \%)$ & 0.669 \\
\hline 2 & $898(89.1 \%)$ & $698(89.7 \%)$ & \\
\hline \multicolumn{4}{|c|}{ Number of high-quality transferred embryos } \\
\hline 0 & $150(14.9 \%)$ & $137(17.6 \%)$ & 0.298 \\
\hline 1 & $258(25.6 \%)$ & $193(24.8 \%)$ & \\
\hline 2 & $600(59.5 \%)$ & $448(57.6 \%)$ & \\
\hline
\end{tabular}

${ }^{a}$ the couples with mild ovarian stimulation were of poor ovarian response. Generally, those couples would have the embryos vitrified for subsequent frozenthawed transfer cycle; ${ }^{b}$ the couples with infertile cause of male factor (azoospermatism) were provided with donor sperm for insemination. 
TABle 2: Clinical outcomes after the first fresh D3 embryo transfer.

\begin{tabular}{lccc}
\hline & Low-TSH $(0.27-2.5 \mathrm{mIU} / \mathrm{L})(n(\%))$ & High-normal TSH $(2.5-4.2 \mathrm{mIU} / \mathrm{L})(n(\%))$ & $P$ \\
\hline Pregnancy rate & $606(60.1)$ & $476(61.2)$ & $452(58.1)$ \\
Clinical pregnancy rate & $568(56.3)$ & $385(49.5)$ & 0.648 \\
Live birth rate & $471(46.7)$ & $24(5)$ & 0.459 \\
Early pregnancy loss rate & $38(6.3)$ & $56(12.4)$ & 0.227 \\
Miscarriage rate & $87(15.3)$ & $1(0.2)$ & 0.388 \\
Still birth rate & $2(0.4)^{\mathrm{a}}$ & $10(2.2)$ & 0.181 \\
Ectopic pregnancy rate & $10(1.8)$ & 0.000 \\
\hline
\end{tabular}

${ }^{\mathrm{a}}$ : two women conceived two babies but gave birth to a singleton.

TABle 3: The possible factors that impact on clinical outcomes.

\begin{tabular}{|c|c|c|c|c|c|c|}
\hline & \multicolumn{2}{|c|}{ Clinical pregnancy } & \multicolumn{2}{|c|}{ Live birth } & \multicolumn{2}{|c|}{ Miscarriage } \\
\hline & $\mathrm{aOR}(95 \% \mathrm{CI})$ & $P$ & $\mathrm{aOR}(95 \% \mathrm{CI})$ & $P$ & $\mathrm{aOR}(95 \% \mathrm{CI})$ & $P$ \\
\hline TSH (low VS high-normal) & $0.92(0.76,1.12)$ & 0.416 & $0.88(0.73,1.08)$ & 0.219 & $1.30(0.90,1.88)$ & 0.163 \\
\hline Age & $0.93(0.91,0.95)$ & $<0.001$ & $0.91(0.89,0.93)$ & $<0.001$ & $1.12(1.08,1.17)$ & $<0.001$ \\
\hline $\mathrm{AMH}$ & $1.00(0.96,1.01)$ & 0.068 & $1.00(0.98,1.01)$ & 0.269 & $1.01(0.98,1.01)$ & 0.181 \\
\hline Number of high-quality embryos & $1.73(1.51,1.99)$ & $<0.001$ & $1.76(1.52,2.03)$ & $<0.001$ & $0.62(0.51,0.86)$ & 0.002 \\
\hline Number of transferred embryos & $1.34(0.96,1.88)$ & 0.085 & $1.31(0.91,1.87)$ & 0.136 & $1.03(0.53,1.99)$ & 0.924 \\
\hline
\end{tabular}

group had live births, while 385 in the high-normal TSH group had live births (Table 4 ). No significant difference was observed in terms of the number of live birth $(P=0.129)$. The preterm birth rates were similar between the two groups (singleton, $P=0.065$; twin, $P=1.000$ ). The low-weight birth rates were also similar between the two groups (singleton, $P=0.679$; twin, $P=1.000)$. In addition, the gestational age and birth length of single live births were similar between the low-TSH and high-normal TSH groups $(P=0.621$ and 0.943 , respectively), as were the gestational age and length of twin live births $(P=0.157$ and 0.904 , respectively).

\section{Discussion}

In this retrospective study, we investigated the association between preconception TSH and the reproductive outcomes of 1786 infertile women undergoing the first fresh D3 embryo transfer at a university-affiliated hospital and found that a high-normal preconception TSH level (between 2.5-4.2 mIU/L) was not associated with lower odds of clinical pregnancy and live birth or higher odds of miscarriage. Neonatal outcomes were also comparable between low-TSH and high-normal TSH groups.

Chen et al. [15] investigated the association of preconception TSH and clinical outcomes in a Chinese woman conceived naturally and clarified that preconceptionally high-TSH levels were associated with a small but significantly increased risk of overall adverse events, even within the normal unpregnant range, and concluded that TSH $<2.5 \mathrm{mIU} / \mathrm{L}$ was more suitable for the assessment of women planning for a pregnancy. However, the impact of preconception TSH in women undergoing fertility treatment is conflicting.

Our previous results were in agreement with other studies, which demonstrated that mildly elevated preconception TSH levels before treatment had no adverse impact on the clinical outcomes in infertile women undergoing intrauterine insemination (IUI) treatment [4]. In addition, we further included a cohort of highly homogeneous infertile women who were transferred with a single fresh D5 blastocyst and found similar reproductive outcomes between the low-TSH and high-normal TSH groups (data not shown). Further on, another cohort of homogeneous infertile women was included and underwent the first D3 embryo transfer in this study. Again, similar results were observed regardless of different embryo transfer schemes and possible inclusion of infertile populations with different age, ovarian reserve, and ovarian stimulation protocol.

Similarly, Reh et al. used different TSH cutoffs in a large retrospective cohort study which included first-cycle IVF patients, and no differences in the rates of clinical pregnancy, delivery, or miscarriage were observed after adjustments were made for age [16]. Aghahosseini et al. reported a lack of association between TSH levels in the range of 0.5-4.5 mIU/ $\mathrm{L}$ and clinical pregnancy rate and stated that lowering the upper limit of normal TSH should still be considered a scientific debate [6]. Chai et al. also reported that the clinical pregnancy rate and miscarriage rate were not impaired when the TSH level was high-normal [8]. In agreement with our results, Unuane et al. and So et al. both claimed that $\mathrm{cu}-$ mulative pregnancy rates were similar between women with low-TSH levels and those with high-normal TSH levels $[5,14]$.

According to the molecular basis of TSH and thyroid hormone action during implantation and early development, thyroid hormone might be involved in endometrium preparation for pregnancy and initial trophoblast development, and thyroid hormones are essential players in the mechanisms regulating implantation and early fetal development [17]. As a matter of fact, FT4 levels between lowTSH and high-normal TSH groups were similar in either our previous studies or the study performed by Unuane et al. 
TABLE 4: The obstetric outcomes of the women undergoing the first fresh D3 embryo transfer.

\begin{tabular}{|c|c|c|c|c|}
\hline & & Low-TSH group $(n=471)$ & High-normal TSH group $(n=385)$ & $P$ \\
\hline \multirow{2}{*}{ Number of live births } & Singleton & $312(62.2 \%)$ & $274(71.2 \%)$ & 0.139 \\
\hline & Twin & $159(33.8 \%)$ & $111(28.8 \%)$ & \\
\hline \multirow{2}{*}{ Birth weight (g) } & Singleton & $3345(575.7)$ & $3390(545.2)$ & 0.334 \\
\hline & Twin & $2527.9(479.1)$ & $2566.5(445.1)$ & 0.342 \\
\hline \multirow{2}{*}{ Birth length $(\mathrm{cm})$} & Singleton & $50.2(2.2)$ & $50.2(2.3)$ & 0.943 \\
\hline & Twin & $48.1(2.7)$ & $48.1(2.6)$ & 0.904 \\
\hline \multirow{2}{*}{ Gestational age (weeks) } & Singleton & $38.9(1.8)$ & $39(1.6)$ & 0.621 \\
\hline & Twin & $36.2(2.2)$ & $36.5(1.6)$ & 0.157 \\
\hline \multirow{2}{*}{ Preterm birth } & Singleton & $36(11.5 \%)$ & $19(6.9 \%)$ & 0.065 \\
\hline & Twin & $73(45.9 \%)$ & $51(45.9 \%)$ & 1.000 \\
\hline \multirow{2}{*}{ Low-weight birth } & Singleton & $14(4.4 \%)$ & $10(3.6 \%)$ & 0.679 \\
\hline & Twin & $51(32.0 \%)$ & $35(31.5 \%)$ & 1.000 \\
\hline
\end{tabular}

[14], which may partially explain the similar results between the two groups.

In contrast, Diana et al. claimed a detrimental effect of high-normal preconception TSH levels on the clinical outcomes of infertile women undergoing their first IVF cycle [10]. Discrepancies might be explained by the differences in the included population and the transferred embryos. Specifically, the proportion of women in the high-normal TSH group was much higher in our study (43.5\% vs. $18.3 \%)$. Notably, the prevalence of SCH (TSH cutoff: $4.2 \mathrm{mIU} / \mathrm{L}$ ) in the general population in China was $16.7 \%$ [18], which was close to the proportion of the population with high-normal TSH levels in the study by Diana et al. In addition, the women in our study were transferred with fresh D3 embryos, while details about the transferred embryos in the study by Diana et al. were not reported. Valerie et al. further demonstrated that a preconception TSH level $>2.5 \mathrm{mIU} / \mathrm{L}$ was associated with a lower gestational age at delivery and lower birth weight in women undergoing IVF treatment [19]. It should be noted that the study performed by Valerie et al. included a population with TSH level $>4.0 \mathrm{mIU} / \mathrm{L}$, which was currently used as the cutoff to define $\mathrm{SCH}$, while our study only included women with TSH values lower than the upper limit of the reference range. The reproductive outcomes which included the incidence of preterm birth rate and low-weight birth rate were comparable when we controlled for twin live births, which supported our observations found in women undergoing their first IUI treatment.

There were also some limitations in this study. First, this was a retrospective study with data collected from a single center, and selection bias was inevitable. Second, we did not report the status of thyroid antibodies in infertile women, which might have a role in infertility and miscarriage. However, on the one hand, the existing data are controversial regarding whether thyroid antibodies are associated with infertility or adverse reproductive outcomes $[7,8,12,14,20]$. On the other hand, according to the two RCTs of high quality, euthyroid women with positive TPO did not benefit from treatment with LT4 $[21,22]$. Third, we included a cohort of infertile women with specified conditions, which we believed to be one of the crucial factors explaining the discrepancies in the results. Whether the results might be applied to women with repeated embryo transfer or healthy women remains to be tested. Finally, we did not monitor the TSH level once it was within the normal range before treatment. It was confirmed in other studies that high-normal TSH was more prone to increase to a level higher than the upper limit of normal range during ovarian stimulation and decrease within the normal range after embryo transfer [23-27]. Whether temporarily increased TSH levels might affect clinical outcomes remained unknown. The strength of our study was the highly homogeneous population-based cohort of euthyroid women, as the number, stage, or quality of transferred embryos, as well as the age and ovarian reserve, might potentially affect the results of embryo transfer. We also compared the differences in obstetric outcomes, such as gestational age, birth weight, and birth length, between the low-TSH and high-normal TSH groups and found that, regardless of TSH levels within the normal range, age and number of high-quality D3 embryos were independently associated with reproductive outcomes such as clinical pregnancy rate, live birth rate, and miscarriage rate.

\section{Conclusion}

We included infertile women as homogeneous as possible to minimize the bias that may lower the power of the impact of TSH on reproductive outcomes. We found that a highnormal TSH level did not significantly influence the reproductive outcomes of infertile women undergoing the first fresh D3 embryo transfer. Further studies are needed to test whether the results might be applicable to a wider population.

\section{Data Availability}

All data generated or analyzed during this study are included in this published article.

\section{Ethical Approval}

This study was approved by the Ethics Committee of the Third Affiliated Hospital of Zhengzhou University. 


\section{Conflicts of Interest}

The authors declare that they have no conflicts of interest.

\section{Authors' Contributions}

WXL and GYC proposed the design ideas. ZYC and WWB checked the data and constructed the tables. ZYC, WWB, and LYL wrote the manuscript. All authors approved the final version of this article. WXL and GYC contributed equally to this paper. Xingling Wang and Yichun Guan contributed equally to this paper.

\section{Acknowledgments}

This work was supported by the grant (LHGJ20190400) from the Joint Project by the Third Affiliated Hospital of Zhengzhou University and Health Commission of Henan Province.

\section{References}

[1] E. K. Alexander, E. N. Pearce, and G. A. Brent, "2017 Guidelines of the American thyroid association for the diagnosis and management of thyroid disease during pregnancy and the postpartum," Thyroid, vol. 27, pp. 315-389, 2017.

[2] G. Mintziori, D. G. Goulis, and E. M. Kolibianakis, "Thyroid function and IVF outcome," Current Opinion in Obstetrics and Gynecology, vol. 28, no. 3, pp. 191-197, 2016.

[3] Practice Committee of the American Society for Reproductive Medicine, "Subclinical hypothyroidism in the infertile female population: a guideline," Fertility and Sterility, vol. 104, pp. 545-553, 2015.

[4] Y. Zhang, W. Wu, and Y. Liu, "The impact of preconceptional serum TSH levels between 2.5 and $4.0 \mathrm{miu} / \mathrm{l}$ on infertile women going through their first iui treatment cycle," International Journal of Endocrinology, vol. 2019, Article ID 8492904, 7 pages, 2019.

[5] S. So, W. Yamaguchi, N. Murabayashi, N. Miyano, and F. Tawara, "Effect of moderately increased thyroid-stimulating hormone levels and presence of thyroid antibodies on pregnancy among infertile women," Reproductive Medicine and Biology, vol. 19, no. 1, pp. 82-88, 2020.

[6] M. Aghahosseini, H. Asgharifard, and A. Aleyasin, "Effects of thyroid stimulating hormone (TSH) level on clinical pregnancy rate via in vitro fertilization (IVF) procedure," Medical Journal of the Islamic Republic of Iran, vol. 28, p. 46, 2014.

[7] D. Unuane, B. Velkeniers, and B. Bravenboer, "2017 Impact of thyroid autoimmunity in euthyroid women on live birth rate after IUI," Human Reproduction, vol. 32, pp. 915-922, 2017.

[8] J. Chai, W. Y. Yeung, and C. Y. Lee, "Live birth rates following in vitro fertilization in women with thyroid autoimmunity and/or subclinical hypothyroidism," Clinical Endocrinology, vol. 80, pp. 122-127, 2014.

[9] A. E. Karmon, M. Batsis, and J. E. Chavarro, "Preconceptional thyroid-stimulating hormone levels and outcomes of intrauterine insemination among euthyroid infertile women," Fertility and Sterility, vol. 103, pp. 258-263, 2015.

[10] D. Grove Laugesen, C. Aaskov, and E. Ebbehøj, "Preconceptional thyrotropin level in euthyroid women is inversely associated with the live birth rate in first in vitro fertilization cycle ACTA," Obstetrics \& Gynecology Ultrasound Scanner Machine, vol. 98, pp. 929-936, 2019.
[11] K. G. Michalakis, T. B. Mesen, and L. M. Brayboy, "Subclinical elevations of thyroid-stimulating hormone and assisted reproductive technology outcomes," Fertility and Sterility, vol. 95, pp. 2634-2637, 2011.

[12] R. Negro, A. Schwartz, R. Gismondi, A. Tinelli, T. Mangieri, and A. Stagnaro-Green, "Increased pregnancy loss rate in thyroid antibody negative women with TSH levels between 2.5 and 5.0 in the first trimester of pregnancy," The Journal of Clinical Endocrinology \& Metabolism, vol. 95, no. 9, pp. E44-E48, 2010.

[13] T. Zhao, B. M. Chen, and X. M. Zhao, "Meta-analysis of ART outcomes in women with different preconception TSH levels," Reproductive Biology and Endocrinology, vol. 1610, p. 1186, 2018.

[14] D. Unuane, B. Velkeniers, and S. Deridder, "Impact of thyroid autoimmunity on cumulative delivery rates in in vitro fertilization/intracytoplasmic sperm injection patients," Fertility and Sterility, vol. 106, pp. 144-150, 2016.

[15] S. Chen, X. Zhou, and H. Zhu, "Preconception tsh and pregnancy outcomes: a population-based cohort study in 184 611 women," Clinical Endocrinology, vol. 86, pp. 816-824, 2017.

[16] A. Reh, J. Grifo, and A. Danoff, "What is a normal thyroidstimulating hormone (TSH) level? effects of stricter tsh thresholds on pregnancy outcomes after in vitro fertilization," Fertility and Sterility, vol. 94, pp. 2920-2922, 2010.

[17] M. Colicchia, L. Campagnolo, and E. Baldini, "Molecular basis of thyrotropin and thyroid hormone action during implantation and early development," Human Reproduction, vol. 20, pp. 884-904, 2014.

[18] Z. Shan, L. Chen, and X. Lian, "Iodine status and prevalence of thyroid disorders after introduction of mandatory universal salt iodization for 16 years in china: a cross-sectional study in 10 Cities," Thyroid, vol. 26, pp. 1125-1130, 2016.

[19] V. L. Baker, H. M. Rone, D. J. Pasta, H. P. Nelson, M. Gvakharia, and G. D. Adamson, "Correlation of thyroid stimulating hormone (TSH) level with pregnancy outcome in women undergoing in vitro fertilization," American Journal of Obstetrics and Gynecology, vol. 194, no. 6, pp. 1668-1674, 2006.

[20] B. Jatzko, E. Vytiska-Bistorfer, and A. Pawlik, "The impact of thyroid function on intrauterine insemination outcome--a retrospective analysis Reprod," Biology and Endocrinology, vol. 12, p. 28, 2014.

[21] H. Wang, H. Gao, and H. Chi, "Effect of levothyroxine on miscarriage among women with normal thyroid function and thyroid autoimmunity undergoing in vitro fertilization and embryo transfer," JAMA, vol. 318, p. 2190, 2017.

[22] R. K. Dhillon-Smith, L. J. Middleton, K. K. Sunner et al., "Levothyroxine in women with thyroid peroxidase antibodies before conception," New England Journal of Medicine, vol. 380, no. 14, pp. 1316-1325, 2019.

[23] C. R. Gracia, C. B. Morse, and G. Chan, "Thyroid function during controlled ovarian hyperstimulation as part of in vitro fertilization," Fertility and Sterility, vol. 97, pp. 585591, 2012.

[24] Y. Du, X. Xin, and N. Cui, "Effects of controlled ovarian stimulation on thyroid stimulating hormone in infertile women," European Journal of Obstetrics \& Gynecology and Reproductive Biology, vol. 234, pp. 207-212, 2019.

[25] K. Poppe, D. Unuane, and M. D’Haeseleer, “Thyroid function after controlled ovarian hyperstimulation in women with and without the hyperstimulation syndrome," Fertility and Sterility, vol. 96, pp. 241-245, 2011. 
[26] L. Benaglia, A. Busnelli, E. Somigliana et al., "Incidence of elevation of serum thyroid-stimulating hormone during controlled ovarian hyperstimulation for in vitro fertilization," European Journal of Obstetrics \& Gynecology and Reproductive Biology, vol. 173, pp. 53-57, 2014.

[27] S. Reinblatt, B. Herrero, J. A. Correa et al., "Thyroid stimulating hormone levels rise after assisted reproductive technology," Journal of Assisted Reproduction and Genetics, vol. 30, no. 10, pp. 1347-1352, 2013. 